\title{
Conductance of a quantum wire at low electron density
}

\author{
K. A. Matveev \\ Argonne National Laboratory, 9700 S Cass Ave., Argonne, IL 60439, USA and \\ Department of Physics, Duke University, Durham, NC 27708, USA
}

(Dated: May 23, 2004)

\begin{abstract}
We study the transport of electrons through a long quantum wire connecting two bulk leads. As the electron density in the wire is lowered, the Coulomb interactions lead to short-range crystalline ordering of electrons. In this Wigner crystal state the spins of electrons form an antiferromagnetic Heisenberg spin chain with exponentially small exchange coupling $J$. Inhomogeneity of the electron density due to the coupling of the wire to the leads results in violation of spin-charge separation in the device. As a result the spins affect the conductance of the wire. At zero temperature the low-energy spin excitations propagate freely through the wire, and its conductance remains $2 e^{2} / h$. Since the energy of the elementary excitations in the spin chain (spinons) cannot exceed $\pi J / 2$, the conductance of the wire acquires an exponentially small negative correction $\delta G \propto-\exp (-\pi J / 2 T)$ at low temperatures $T \ll J$. At higher temperatures, $T \gg J$, most of the spin excitations in the leads are reflected by the wire, and the conductance levels off at a new universal value $e^{2} / h$.
\end{abstract}

PACS numbers: 73.63.Nm, 73.21.Hb, 75.10.Pq

\section{INTRODUCTION}

The quantization of conductance of one-dimensional (1D) electron systems in units of $2 e^{2} / h$ was first observed in experiments with quantum point contacts 1.2 The latter consist of a short (well under $1 \mu \mathrm{m}$ ) 1D constriction connecting two bulk two-dimensional leads. Further progress in fabrication of low-disorder devices resulted in observation ${ }^{3,4}$ of similar conductance quantization in quantum wires of several microns in length. Experimentally the quantization is observed as very flat plateaus in the dependence of linear conductance on the voltage at the gate controlling the electron density in the wire.

In a number of recent experiments $5,6,7,8,9,10,11,12,13$ deviations of conductance from perfect quantization have been observed at low electron density. These deviations manifest themselves as negative corrections to the conductance at the beginning of the first quantized plateau. The correction is usually small at the lowest temperatures available, but becomes significant at $T \sim 1 \mathrm{~K}$. In typical samples 5.6 .7 the conductance levels off at high temperatures and forms a quasi-plateau at about $0.7 \times\left(2 e^{2} / h\right)$. This phenomenon is often referred to as the 0.7 structure. Despite the numerous theoret-

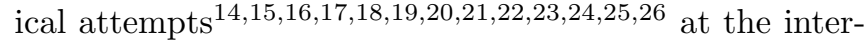
pretation of the 0.7 structure, its origin remains unclear.

The analysis of the existing experimental data shows that the 0.7 structure is sensitive to the length of the one-dimensional region connecting the two-dimensional leads. The structure tends to be relatively weak in the short contacts,, 8.9 where no quasi-plateau is observed even at high temperatures. On the other hand, in longer samples $10,11,12,13$ the plateau is observed even at the lowest temperatures available. The effect is also somewhat stronger, with the quasi-plateau moving to a lower value of conductance $G \approx 0.5 \times\left(2 e^{2} / h\right)$.
In this paper we consider conductance of a long quantum wire in the regime of low electron density $n$. Focusing on the case of a GaAs device, we assume quadratic energy spectrum for free electrons $\epsilon(p)=p^{2} / 2 m$, where $m$ is the effective mass of the electrons. The typical kinetic energy of an electron at zero temperature is of the order of the Fermi energy, $E_{F}=(\pi \hbar n)^{2} / 8 m$. It is important to note that at low density $n \ll a_{B}^{-1}$ the kinetic energy is small compared to the typical energy $e^{2} n / \varepsilon$ of Coulomb interaction between electrons, where $\varepsilon$ is the dielectric constant, and $a_{B}=\varepsilon \hbar^{2} / m e^{2}$ is the effective Bohr radius of the material. Thus in the limit of low density the electrons can be viewed as classical particles placed at equidistant positions to minimize the Coulomb repulsion. Such a picture was first proposed by Wigner ${ }^{27}$ and will be referred to as the Wigner crystal.

Although the quantum fluctuations of electrons near their equilibrium positions destroy the long-range order in the Wigner crystal, its short-range structure strongly affects the transport through the wire. In particular, the electrons occupying well-defined sites of a Wigner lattice can be viewed as an antiferromagnetic spin chain with exponentially small exchange constant $J$. The appearance of a new energy scale $J \ll E_{F}$ significantly affects the physics of the electronic transport through the wire. This effect is most important at intermediate temperatures, $J \ll T \ll E_{F}$, where it results in a considerable suppression of the conductance of the wire.

The physics of this phenomenon is controlled by the effect of spin-charge separation in one-dimensional interacting electron systems. The latter refers to the fact that fermionic quasiparticles cannot be viewed as elementary excitations of the system, i.e., it no longer behaves as a Fermi liquid. Instead, the system displays Luttinger liquid behavior, and the elementary excitations are bosonic waves of charge and spin densities propagating at dif- 
ferent velocities. As a result, when an electron enters the one-dimensional region from one of the leads, it is decomposed into charge and spin waves. At low temperature $T \ll J$ both waves pass through the wire, and upon reaching the other lead they reassemble into a fermionic quasiparticle. This process can be interpreted as perfect transmission of electrons through the wire and gives the standard value of conductance $G=2 e^{2} / h$. On the other hand, at $T \gg J$, the bandwidth $\sim J$ of the spin excitations in the wire is small compared to their typical energy $\sim T$. As a result, only the charge excitations pass through the wire, whereas the spin ones are reflected back to the lead. We show below that this additional scattering of spin excitations by the wire reduces its conductance to $e^{2} / h$

In Sec. III we study the applicability of the Luttinger liquid description to the 1D Wigner crystal and show that it is only valid at energy scales below $J$. On the other hand, the property of spin-charge separation is more general and persists at energy scales above $J$. We review the known results for the conductance of a quantum wire in Sec. III The most important consequence of the spincharge separation in quantum wires is the conclusion that spin degrees of freedom do not affect the conductance, which remains quantized at $2 e^{2} / h$. In Sec. IV we show that the spin-charge separation is violated when the wire is connected to the leads. As a result the spin subsystem affects the propagation of electric charge through the wire and contributes to its resistance. This contribution is studied in Sec. $\mathbb{\nabla}$ where we find that at $J \ll T$ the conductance of the device reduces from $2 e^{2} / h$ to $e^{2} / h$. The relation of our results to experimental measurements of conductance of quantum wires is discusses in Sec. VI A brief summary of some of our results has been reported in Ref. 29 .

\section{SPIN-CHARGE SEPARATION IN QUANTUM WIRES}

It has been known since the seventies ${ }^{30}$ that the lowenergy excitations of a 1D system of interacting electrons are the charge and spin waves propagating independently of each other at different velocities. This result is valid at the energy scales low compared to the bandwidths $D_{\rho}$ and $D_{\sigma}$ of the charge and spin excitations. In the case of not very strong interactions both bandwidths are of the order of the Fermi energy, and the picture of completely separated charge and spin excitations is appropriate at $T \ll E_{F}$.

At low electron density $n \ll a_{B}^{-1}$ the interactions between electrons are strong. We show below that as a result the velocity of the spin excitations is greatly reduced, and their bandwidth $D_{\sigma} \sim J$ becomes much smaller than $E_{F}$. A similar effect is known to occur in the strong interaction limit of some lattice models, such as the Hubbard model ${ }^{31}$ In this regime, the description of the spin excitations in the language of non-interacting spin waves is applicable only at very low temperatures $T \ll J$. We show in this section that a generalized picture of decoupled charge and spin excitations remains valid even at $T \gtrsim J$. In this picture the charge excitations are still given by the waves of charge density (plasmons), and the spin waves are replaced with the excitations of a Heisenberg spin chain.

\section{A. Luttinger-liquid picture of one-dimensional electrons systems}

The problems involving low-energy properties of interacting 1D electron systems are conveniently described in the framework of the bosonization technique ${ }^{28.32}$ The first step in this approach is to linearize the spectrum of electrons near the Fermi level, thereby replacing the quadratic dispersion law $\epsilon(k)=\hbar^{2} k^{2} / 2 m$ with the linear one. In this Tomonaga-Luttinger model the electrons are separated in two branches, the left- and right-movers, with energies $\epsilon_{L, R}(k)=\hbar v_{F}\left(\mp k-k_{F}\right)$, where $v_{F}$ and $k_{F}$ are the Fermi velocity and Fermi wavevector. One can then present the fermionic field operators $\psi_{L, \lambda}$ and $\psi_{R, \lambda}$ in terms of fields $\phi_{\lambda}$ and $\theta_{\lambda}$ satisfying bosonic commutation relations $\left[\phi_{\lambda}(x), \partial_{y} \theta_{\lambda^{\prime}}(y)\right]=i \pi \delta(x-y) \delta_{\lambda \lambda^{\prime}}$ using the following rule

$$
\begin{aligned}
\psi_{L, \lambda}(x) & =\frac{\eta_{L, \lambda}}{\sqrt{2 \pi \alpha}} e^{-i k_{F} x} e^{i \phi_{\lambda}(x)-i \theta_{\lambda}(x)}, \\
\psi_{R, \lambda}(x) & =\frac{\eta_{R, \lambda}}{\sqrt{2 \pi \alpha}} e^{i k_{F} x} e^{-i \phi_{\lambda}(x)-i \theta_{\lambda}(x)} .
\end{aligned}
$$

Here $\lambda=\uparrow, \downarrow$ is the spin index, $\alpha$ is the short distance cutoff, $\eta_{L, \lambda}$ and $\eta_{L, \lambda}$ are Majorana fermion operators ${ }^{32}$

In terms of the bosonic variables the Hamiltonian of an interacting 1D electron system takes the form ${ }^{32.33}$

$$
H=H_{\rho}+H_{\sigma}
$$

where the two terms $H_{\rho}$ and $H_{\sigma}$ describe the excitations of the charge and spin degrees of freedom, respectively, and have the forms

$$
\begin{aligned}
H_{\rho}= & \int \frac{\hbar u_{\rho}}{2 \pi}\left[\pi^{2} K_{\rho} \Pi_{\rho}^{2}+K_{\rho}^{-1}\left(\partial_{x} \phi_{\rho}\right)^{2}\right] d x, \\
H_{\sigma}= & \int \frac{\hbar u_{\sigma}}{2 \pi}\left[\pi^{2} K_{\sigma} \Pi_{\sigma}^{2}+K_{\sigma}^{-1}\left(\partial_{x} \phi_{\sigma}\right)^{2}\right] d x \\
& +\frac{2 g_{1 \perp}}{(2 \pi \alpha)^{2}} \int \cos \left[\sqrt{8} \phi_{\sigma}(x)\right] d x .
\end{aligned}
$$

Here the new bosonic fields $\phi_{\rho, \sigma}=\left(\phi_{\uparrow} \pm \phi_{\downarrow}\right) / \sqrt{2}$ and $\Pi_{\rho, \sigma}=\partial_{x}\left(\theta_{\uparrow} \pm \theta_{\downarrow}\right) / \pi \sqrt{2}$ satisfy the standard commutation relations $\left[\phi_{\alpha}(x), \Pi_{\alpha^{\prime}}(y)\right]=i \delta(x-y) \delta_{\alpha \alpha^{\prime}}$ and represent the excitations of the charge and spin degrees of freedom. The Hamiltonian (2)-(4) depends on five parameters determined by the interactions between electrons: velocities $u_{\rho}$ and $u_{\sigma}$ of the charge and spin excitations, dimensionless parameters $K_{\rho}$ and $K_{\sigma}$, and the matrix element $g_{1 \perp}$ of spin-flip scattering of a left-moving 
electron and a right-moving one. In the absence of interactions, $u_{\rho}=u_{\sigma}=v_{F}, K_{\rho}=K_{\sigma}=1$, and $g_{1 \perp}=0$. The bosonized Hamiltonian correctly describes the charge and spin excitations of the system with energies below the respective bandwidths $D_{\rho, \sigma} \sim \hbar n u_{\rho, \sigma}$.

In the most interesting case of repulsive interactions $K_{\rho}<1$. The coupling constant $g_{1 \perp}$ is positive and scales to zero at low energy scales $D$,

$$
g_{1 \perp}=\frac{g_{1 \perp}}{1+\frac{g_{1 \perp}}{\pi u_{\sigma}} \ln \frac{D_{\sigma}}{D}} .
$$

The parameter $K_{\sigma}$ renormalizes along with $g_{1 \perp}$, approaching the value $K_{\sigma}=1$ required by the $\mathrm{SU}(2)$ symmetry as $K_{\sigma}=1+g_{1 \perp} / 2 \pi u_{\sigma}$, Ref. 3233 .

Since the sine-Gordon term in Eq. (4) vanishes at $D / D_{\sigma} \rightarrow 0$, the Hamiltonian (2) becomes quadratic and describes a Luttinger liquid. ${ }^{28}$ The latter represents a stable fixed point of the problem, so the description of the system based upon the Hamiltonian (2)-(4) is expected to be valid in a broad range of interaction strengths. It is not immediately obvious, however, that the above picture is applicable at low electron density $n$, i.e., when the interactions are so strong that the electrons form a Wigner crystal. Indeed, a Fourier expansion of the wavefunction of an electron localized in a small region of size $a \ll n^{-1}$ near a given lattice site involves the wavevectors in a broad range $\delta k \sim 1 / a \gg n \sim k_{F}$. Thus the standard procedure of linearization of the electronic spectrum near $k_{F}$ leading to the Tomonaga-Luttinger model is not justified in this case. In addition, each electron is constructed out of waves with both positive and negative wavevectors, and the picture of two separate branches of left- and right-moving particles is not applicable to the Wigner crystal. We show now that even though the conventional derivation leading to Eqs. (2) -(4) is not justified, this Hamiltonian does describe the low-energy properties of a 1D Wigner crystal.

\section{B. Charge and spin excitations in a Wigner crystal}

At low electron density, $n a_{B} \ll 1$, the properties of the system are dominated by the Coulomb repulsion, and the electrons occupy fixed positions on the Wigner lattice. The first correction to this picture is due to the small vibrations of the lattice, analogous to phonons in conventional crystals. In the long-wavelength limit these phonons can be described in the framework of elasticity theory. In this approach the crystal is viewed as an elastic medium. Its motion is described in terms of the displacement $u(x)$ of the medium at point $x$ from its equilibrium position and the momentum density $p(x)$. The energy of the system can then be written as a sum of kinetic and potential energies,

$$
H=\int\left[\frac{p^{2}}{2 m n}+\frac{1}{2} m n s^{2}\left(\partial_{x} u\right)^{2}\right] d x .
$$

The second term here is $\frac{1}{2}\left(\partial^{2} E / \partial n^{2}\right)(\delta n)^{2}$, where $E$ is the energy of the resting medium per unit length, and the density perturbation $\delta n$ is proportional to the deformation of the medium, $\delta n=-n \partial_{x} u$. The parameter $s=\sqrt{(n / m)\left(\partial^{2} E / \partial n^{2}\right)}$ has the meaning of the speed of density waves (plasmons) in the Wigner crystal.

The speed of plasmons in a $1 \mathrm{D}$ system with true Coulomb interactions between electrons diverges in the limit of long wavelength. In practice, however, the interactions between electrons are usually screened at large distances by a remote metal gate. In the model where the gate is a conducting plane at a large distance $d \gg n^{-1}$ from the Wigner crystal, the speed of plasmons is

$$
s=\sqrt{\frac{2 e^{2} n}{\varepsilon m} \ln (\zeta n d)}
$$

where $\zeta \approx 8.0$, Ref. 34 .

The classical Hamilton function (6) can be quantized by imposing commutation relations $[u(x), p(y)]=i \hbar \delta(x-$ $y)$. The resulting Hamiltonian describes the propagation of the electron density excitations in a Wigner crystal, and is completely analogous to the term $H_{\rho}$ in the Hamiltonian (2) - (4) of the Luttinger liquid. Comparing the commutation relations of the bosonic fields entering Hamiltonians (3) and (6), and taking into account the expressions for the density perturbation $\delta n=-\frac{\sqrt{2}}{\pi} \partial_{x} \phi_{\rho}=$ $-n \partial_{x} u$, we identify the fields as

$$
u(x)=\frac{\sqrt{2}}{\pi n} \phi_{\rho}(x), \quad p(x)=\frac{\pi n \hbar}{\sqrt{2}} \Pi_{\rho}(x) .
$$

Using these expressions we can relate the parameters in the Hamiltonian $H_{\rho}$ to the properties of the Wigner crystal as follows

$$
u_{\rho}=s, \quad K_{\rho}=\frac{v_{F}}{s} .
$$

Here $v_{F}=\pi \hbar n / 2 m$ is the Fermi velocity in a noninteracting Fermi gas of density $n$.

The electrons in a Wigner crystal are repelled from each other by strong Coulomb forces. In the harmonic chain approximation we used so far the electrons are allowed to move about their equilibrium positions; however, the amplitude of these oscillations remains small. As a result the electrons never move from one site on the Wigner lattice to another, and can be viewed as distinguishable particles. Therefore the energy of the Wigner crystal state in this approximation does not depend on the electron spins.

To account for the spin dependence, one has to include the processes in which electrons tunnel through the Coulomb potential repelling them. Considering a pair of electrons at two neighboring sites of the Wigner lattice, one notices that depending on their total spin, the two electrons occupy either a symmetric or an antisymmetric state in the respective double-well potential. Thus the energy of the pair contains a term $J \boldsymbol{S}_{1} \cdot \boldsymbol{S}_{2}$, where $J>0$ is the difference of energies of the antisymmetric 
and symmetric states, and $\boldsymbol{S}_{i}$ are the operators of electron spins 35 Taking into account all the nearest neighbor sites, we find that the spin properties of the Wigner crystal are described by the Hamiltonian of an antiferromagnetic Heisenberg spin chain

$$
H_{\sigma}=\sum_{l} J \boldsymbol{S}_{l} \cdot \boldsymbol{S}_{l+1}
$$

Since the exchange is due to tunneling, the constant $J$ is exponentially small,

$$
J=J^{*} \exp \left(-\frac{\eta}{\sqrt{n a_{B}}}\right) .
$$

To accurately evaluate $J$ one has to take into account the fact that when two neighboring electrons tunnel through the Coulomb barrier separating them, all other electrons also move. Häusler ${ }^{36}$ suggested an approximation that neglects the motion of other electrons; his result corresponds to the value of $\eta \approx 2.87$ in a finite chain of 15 electrons. We solve this model in the case of an infinite chain in Appendix $\mathrm{A}$ and obtain the value of $\eta \approx 2.82$. We also estimate the prefactor as

$$
J^{*} \approx 1.79 \frac{E_{F}}{\left(n a_{B}\right)^{3 / 4}},
$$

where $E_{F}=(\pi \hbar n)^{2} / 8 m$ is the Fermi energy of a noninteracting electron gas of density $n$.

The spin part (10) of the Hamiltonian of a 1D Wigner crystal is very different from that of a weakly interacting electron gas, Eq. (4). It is easy to show, however, that at low energies $D \ll J$ the two Hamiltonians are equivalent. To accomplish that we use the standard procedure ${ }^{32.33}$ of bosonization of spin chains. The first step is to perform the Jordan-Wigner transformation

$$
S_{l}^{z}=a_{l}^{\dagger} a_{l}-\frac{1}{2}, \quad S_{l}^{x}+i S_{l}^{y}=a_{l}^{\dagger} \exp \left(i \pi \sum_{j=1}^{l-1} a_{j}^{\dagger} a_{j}\right)
$$

which expresses the spin operators in terms of creation and destruction operators $a^{\dagger}$ and $a$ of spinless fermions. In terms of these operators the Hamiltonian (10) becomes

$$
\begin{aligned}
H_{\sigma}= & \frac{1}{2} \sum_{l} J\left[\left(a_{l}^{\dagger} a_{l+1}+a_{l+1}^{\dagger} a_{l}\right)\right. \\
& \left.+2\left(a_{l}^{\dagger} a_{l}+\frac{1}{2}\right)\left(a_{l+1}^{\dagger} a_{l+1}+\frac{1}{2}\right)\right] .
\end{aligned}
$$

Thus the Heisenberg spin chain (10) is equivalent to the model (14) of interacting lattice fermions.

The second step is to bosonize the Hamiltonian (14). At low energies one can replace the lattice model (14) with a continuous one, $a_{l} \rightarrow a(y)$, linearize the spectrum of the fermions near the Fermi level, and then apply a bosonization transformation

$$
a_{L, R}(y)=\frac{\eta_{L, R}}{\sqrt{2 \pi \alpha}} e^{\mp i k_{F} y} e^{ \pm i \phi_{\sigma}(y) / \sqrt{2}-i \sqrt{2} \theta_{\sigma}(y)} .
$$

The resulting bosonized Hamiltonian of the spin chain is equivalent ${ }^{60}$ to Eq. (4). The value of the speed $u_{\sigma}$ of the spin excitations is easily deduced from the Bethe ansatz solution ${ }^{37.38}$ of the Heisenberg model,

$$
u_{\sigma}=\frac{\pi J}{2 \hbar n}
$$

Thus we have established that the bosonized Hamiltonian (2) - (4) adequately describes the low-energy properties of not only weakly interacting electron systems, but also of a $1 \mathrm{D}$ Wigner crystal state at $n a_{B} \ll 1$. However, it is important to keep in mind that the applicability of the bosonized description to the Wigner crystal is limited to very low temperatures $T \ll J$. Given the exponential dependence (11) of the exchange constant on density, this condition can be easily violated even at fairly low temperatures. In this case one has to use the more complicated form (10) of the Hamiltonian $H_{\sigma}$. We show in Sec. V that this breakdown of the Luttinger liquid picture gives rise to significant deviations of conductance of quantum wires from the quantized value $2 e^{2} / h$.

\section{Spin-charge separation at ultralow electron densities}

The Wigner crystal picture discussed in Sec. IB relies on the long-range nature of the Coulomb interaction potential $V(x)=e^{2} / \varepsilon|x|$. In general, a 1D electron system forms a Wigner crystal state at $n \rightarrow 0$ only if the interaction potential decays slower than $1 /|x|^{2}$ at $x \rightarrow \infty$. Indeed, for potential $V(x) \propto 1 /|x|^{\gamma}$ the interaction energy of two electrons at the typical interparticle distance $n^{-1}$ is $V \propto n^{\gamma}$, whereas the kinetic energy $E_{F} \propto n^{2}$. Thus at $\gamma>2$ the interaction energy is negligible at $n \rightarrow 0$.

The electron density in quantum wires is usually controlled by applying voltage to metal gates. The presence of a gate affects the electron-electron interactions at large distances. For instance, if the gate is modeled by a conducting plane at a distance $d$ from the wire, the interactions between electrons become

$$
V(x)=\frac{e^{2}}{\varepsilon}\left(\frac{1}{|x|}-\frac{1}{\sqrt{x^{2}+(2 d)^{2}}}\right) .
$$

The screening of the Coulomb potential by the gate reduces the potential (17) to $V(x)=2 e^{2} d^{2} / \varepsilon|x|^{3}$ at large $|x|$. Therefore, the Wigner crystal picture fails in the limit $n \rightarrow 0$. Comparing the interaction potential at the interparticle distance $V\left(n^{-1}\right)$ with the Fermi energy of electrons, one concludes that within the model (17) the Wigner crystal state exists only in the density range $a_{B} / d^{2} \ll n \ll a_{B}^{-1}$.

As long as the system is in the Wigner crystal state, its spin excitations are described by the Heisenberg model (10). However, the expression (11) for the coupling constant $J$ relies on the pure Coulomb interaction between electrons. In the case of interaction potential screened by 
the gate the exponential decrease of $J$ with decreasing density stops at $n \sim d^{-1}$, because the potential screened by the gates falls off rapidly at distances $x \gg d$. Using the method described in Appendix $\mathrm{A}$ one estimates

$$
J \sim E_{F}\left(\frac{n d^{2}}{a_{B}}\right)^{3 / 4} \exp \left(-\tilde{\eta} \sqrt{\frac{d}{a_{B}}}\right)
$$

at $a_{B} / d^{2} \ll n \ll d^{-1}$. In the case of interaction potential (17) the constant $\tilde{\eta} \approx 8.49$.

The distance to the gate in quantum wire devices is typically large, $d \gtrsim 10 a_{B}$, and most experiments are performed at densities well above $a_{B} / d^{2}$. However, if the density is reduced to $n \lesssim a_{B} / d^{2}$, the Wigner crystal picture used in Sec. IIB will fail. It is interesting to explore to what extent the conclusions of Sec. IIB will be affected. To this end, let us now study the limit $n d^{2} / a_{B} \rightarrow 0$.

At $n \ll a_{B} / d^{2}$ the interaction between two particles at a typical distance $n^{-1}$ is small compared to their kinetic energy $\sim E_{F}$. On the other hand, when the distance between electrons is sufficiently short, $|x| \lesssim$ $n^{-1}\left(n d^{2} / a_{B}\right)^{1 / 3} \ll n^{-1}$, they experience strong repulsion $V(x) \gtrsim E_{F}$. Thus in the limit of low electron density one can model the interaction potential (17) by short-range repulsion

$$
V(x)=\mathcal{V} \delta(x) .
$$

The constant $\mathcal{V}$ should be chosen in such a way that the scattering phase shift for two electrons interacting with potentials $V(x)$ and $\mathcal{V} \delta(x)$ are identical. For the model (17) this condition gives

$$
\mathcal{V} \sim \frac{\hbar^{2} a_{B}}{m d^{2}} \exp \left(\tilde{\eta} \sqrt{\frac{d}{a_{B}}}\right) .
$$

The exponentially large value of $\mathcal{V}$ reflects the fact that the strong repulsion (17) leads to almost perfect backscattering of electrons off each other.

At $\mathcal{V} \rightarrow \infty$ the electrons are separated by thin hardcore potentials. In this limit they can be viewed as distinguishable particles, and the eigenvalues of energy become independent of the electron spins. The wavefunctions of the system essentially coincide with the Slater determinants for spinless non-interacting fermions. Upon bosonization, the Hamiltonian $H_{\rho}$ of this system takes the form (31). The plasmon velocity $s$ in this system is the Fermi velocity of non-interacting electron gas of density $n$, which is twice the Fermi velocity of non-polarized electron gas, $s=2 v_{F}$. Thus according to Eq. (9) we have $K_{\rho}=1 / 2$. Additional properties of this model were recently discussed in Refs. 3940 .

At large finite $\mathcal{V}$ the electrons can change places as a result of scattering, and the energy acquires a weak dependence on the spins. This dependence can be deduced from the well-known properties of the one-dimensional Hubbard model. It has been shown by Ogata and
Shiba ${ }^{41.42}$ that at $U / t \rightarrow \infty$ the spin and charge excitations of the Hubbard model are completely separated, with the Hamiltonian of spin excitations taking the form of the Heisenberg model (10). The magnitude of the exchange constant in this Hamiltonian was found ${ }^{42}$ to be

$$
J=\frac{4 t^{2}}{U} n_{e}\left(1-\frac{\sin 2 \pi n_{e}}{2 \pi n_{e}}\right)
$$

where $n_{e}$ is the average number of electrons per site. In the limit $n_{e} \rightarrow 0$ the Hubbard model is equivalent to an electron gas with quadratic spectrum and point-like interaction (19). The limiting procedure can be performed by introducing infinitesimal lattice period $a$ in the Hubbard model, identifying the parameters $t=\hbar^{2} / 2 m a^{2}$, $U=\mathcal{V} / a, n_{e}=n a$, and taking the limit $a \rightarrow 0$. Applying this procedure to the formula (21), we find

$$
J=\frac{2 \pi^{2}}{3} \frac{\hbar^{4} n^{3}}{m^{2} \mathcal{V}}
$$

Using the estimate (20) of parameter $\mathcal{V}$ for the interaction potential (17), we find

$$
J \sim E_{F} \frac{n d^{2}}{a_{B}} \exp \left(-\tilde{\eta} \sqrt{\frac{d}{a_{B}}}\right) .
$$

Note that the our results (18) and (23) for the exchange constant are of the same order of magnitude at $n=a_{B} / d^{2}$.

So far we have demonstrated that the description of the system in terms of the Hamiltonian in spin-charge separated form $H=H_{\rho}+H_{\sigma}$, with $H_{\rho}$ and $H_{\sigma}$ given by Eqs. (3) and (10) is valid in two different regimes. The first one is the Wigner crystal state at electron densities in the range $a_{B} / d^{2} \ll n \ll a_{B}^{-1}$, and the second is the low density limit $n \ll a_{B} / d^{2}$, where the picture of point-like interactions (19) is applicable. One can show $\underline{43}$ that in fact this picture of spin-charge separation holds at any density $n \ll a_{B}^{-1}$, including the regime $n \sim a_{B} / d^{2}$.

The exchange constant $J$ in the effective spin chain Hamiltonian (10) monotonically decreases as the electron density $n$ is lowered. In the most interesting range of densities $d^{-1} \ll n \ll a_{B}^{-1}$ the dependence of exchange on $n$ is exponential, Eq. (111). At lower densities the dependence becomes a power-law one. Specifically, in the density ranges $a_{B} / d^{2} \ll n \ll d^{-1}$ and $n \ll a_{B} / d^{2}$ one can use the estimates (18) and (23), respectively.

For the sake of simplicity, in the following sections we assume that the electron density is in the range $a_{B} / d^{2} \ll n \ll a_{B}^{-1}$, and refer to the electron system as a Wigner crystal. However, all of our conclusions remain valid at any densities $n \ll a_{B}^{-1}$, if the value of the exchange constant $J$ is adjusted as discussed in this section. 


\section{CONDUCTANCE OF A QUANTUM WIRE WITH SPIN-CHARGE SEPARATION}

The spin-charge separation has a profound effect on the conductance of quantum wires. Indeed, the electric field applied to the wire couples to the electron charges and has no effect on spins. As a result, the spin degrees of freedom remain decoupled from charge ones, and the rather complex form of the Hamiltonian $H_{\sigma}$ has no effect on the conductance. In this section we review the known results for the conductance of a quantum wire with spincharge separation.

\section{A. Infinite wire}

Conductance of an infinite Luttinger liquid is given by $G=2 K_{\rho} e^{2} / h$. This result was obtained ${ }^{44.45}$ by assuming that a weak electric field is applied to a small part of the wire, and the conductance was evaluated by using the Kubo formula. In the following sections it will be more convenient to evaluate the conductance of the Wigner crystal in the regime of applied current. It is therefore instructive to reproduce the result $G=2 K_{\rho} e^{2} / h$ in this approach.

Let us consider a quantum wire whose charge dynamics are described by the Hamiltonian (31), and enforce the current $I=I_{0} \cos \omega t$ at $x=0$. By doing so we impose a boundary condition upon the charge field $\phi_{\rho}(0, t)$. Indeed, the bosonization expression for the electric current is $I=e \frac{\sqrt{2}}{\pi} \dot{\phi}_{\rho}$. (In the case of a Wigner crystal, this can be checked by using Eq. (8) and the definition $I=e n \dot{u}$ of current in terms of the velocity $\dot{u}$ of the crystal.) Thus the field $\phi_{\rho}$ satisfies the condition

$$
\phi_{\rho}(0, t)=\frac{\pi}{\sqrt{2}} q(t)
$$

where the function

$$
q(t)=\frac{I_{0}}{e \omega} \sin \omega t .
$$

is related to the current as $I=e \dot{q}$ and has the meaning of the number of electrons that passed through point $x=0$ at time $t$.

By imposing a time-dependent boundary condition (24) we drive the system with an external oscillating force. This leads to emission of plasmon waves and dissipation of the energy from the driving force to the infinite Luttinger liquid. We will find the resistance of the wire $R_{\rho}$ by evaluating the energy $W$ dissipated in unit time and comparing the result with the Joule heat law $W=\frac{1}{2} I_{0}^{2} R_{\rho}$. We present a formal derivation in Appendix $[\mathrm{B}$ here we limit ourselves to a simple semiclassical argument.

Solving the Hamilton equations with Hamiltonian (3) and boundary condition (24) we find ${ }^{62}$

$$
\begin{aligned}
\phi_{\rho}(x, t) & =\frac{\pi I_{0}}{\sqrt{2} e \omega} \sin \omega\left(t-|x| / u_{\rho}\right), \\
\Pi_{\rho}(x, t) & =\frac{I_{0}}{\sqrt{2} e K_{\rho} u_{\rho}} \cos \omega\left(t-|x| / u_{\rho}\right) .
\end{aligned}
$$

Substituting this solution back into Eq. (3), we find the following expression for the time-averaged energy density in the Luttinger liquid,

$$
\langle E\rangle_{t}=\frac{\pi \hbar}{4 e^{2}} \frac{I_{0}^{2}}{K_{\rho} u_{\rho}} .
$$

The plasmon wave (26) carries the energy $\langle E\rangle_{t}$ at speed $u_{\rho}$ in two directions. Thus the total energy dissipated into plasmon waves in unit time is given by $W=2 u_{\rho}\langle E\rangle_{t}$. Comparing this result with $W=\frac{1}{2} I_{0}^{2} R_{\rho}$, we find the resistance

$$
R_{\rho}=\frac{h}{2 K_{\rho} e^{2}},
$$

in agreement with the result for the conductance found in Ref. 44.45 .

\section{B. Finite-length quantum wire between two non-interacting leads}

The result $G=2 K_{\rho} e^{2} / h$ indicates that in a quantum wire with repulsive interactions conductance should be below the quantized value $2 e^{2} / h$. Furthermore, it is expected to decrease as the electron density $n$ is lowered. However, the experiments consistently show perfect quantization ${ }^{63}$ of conductance at $2 e^{2} / h$ in a broad range of $n$.

This controversy was resolved 46.47 .48 by noticing that instead of an infinite quantum wire, the experiments study transport through a finite-length wire connecting two bulk leads. Since the leads are not one-dimensional, their properties are not adequately described by the Luttinger liquid model (2)-(4). Instead, the electrons in the leads are expected to be in a Fermi liquid state.

To find the conductance of such devices, one can mode ${ }^{46,47.48}$ the leads connected to the wire by two semiinfinite non-interacting wires. In this model the system remains one-dimensional, but the interactions are nonvanishing only in the central part of the system. The length $L$ of the interacting part is identified with the length of the wire. Assuming that the interactions fall off gradually at $x \rightarrow \pm \infty$, one can neglect the backscattering of electrons from the interacting region. In this limit the charge dynamics are still described by the Hamiltonian (3), but the parameters $K_{\rho}$ and $u_{\rho}$ become functions of coordinate $x$.

The measurements of dc conductance in experiments are conducted at very low frequencies $\omega \ll u_{\rho} / L$. Thus the wavelength of the plasmons emitted in the system is 
much greater than the length of the interacting region $L$. Consequently, one should use the value of the parameter $K_{\rho}$ in Eq. (28) taken at $x \rightarrow \pm \infty$, i.e., in the non-interacting region, where $K_{\rho}=1$. Thus the resistance (28) of the device becomes

$$
R_{\rho}=\frac{h}{2 e^{2}}
$$

restoring the perfect quantization. Careful treatments ${ }^{46,47,48}$ of the problem lead to the same conclusion.

\section{VIOLATION OF SPIN-CHARGE SEPARATION IN QUANTUM WIRE DEVICES}

As we saw in Sec. III the inhomogeneity of the system caused by coupling of the wire to the leads changes the conductance from $2 K_{\rho} e^{2} / h$ to $2 e^{2} / h$. This conclusion was derived from consideration of the charge excitations only, as the spin degrees of freedom were assumed to be completely separated. We now turn to the effect of the inhomogeneity on the spin part $H_{\sigma}$ of the Hamiltonian.

We assume that the central part of the wire contains a purely one-dimensional electron system at low density $n \ll a_{B}^{-1}$, so that the Wigner crystal model is appropriate. The wire is also assumed to be smoothly connected to the leads, where the effective interactions are weak. This is due to several effects. First, the electron density grows as one moves away from the wire into the leads. This effectively reduces the interaction strength, as the latter is characterized by parameter $\left(n a_{B}\right)^{-1}$. In addition, the wire becomes wider when it couples to the leads. As a result, when two electrons arrive at the same coordinate $x$ along the wire, they are no longer as close to each other as in the middle of the wire. This reduces the strength of interactions between 1D electrons. The two mechanisms have very similar effect on the Hamiltonian $H_{\sigma}$. For simplicity, in the following we limit our discussion to the effect of inhomogeneous electron density.

Following the ideas of Refs. 46 47.48, we model the wire connected to the leads by an inhomogeneous 1D system. The main source of inhomogeneity is the dependence $n(x)$ of the electron density on position. We assume that the density takes a constant value $n(x)=n$ inside the wire, i.e., at $|x|<L / 2$, and gradually grows to a very large value $n_{\infty} \gg a_{B}^{-1}$ at $x \rightarrow \pm \infty$.

In experimental devices the dependence of electron density on the coordinate along the wire is caused by inhomogeneity of the external confining potential. Apart from changing the electron density, the external potential may also lead to backscattering of electrons in the wire. In a sufficiently long wire such processes may greatly suppress the conductance at low temperature 44.45 In the Wigner crystal picture this phenomenon is interpreted as pinning of the crystal by the external potential. ${ }^{34}$ On the other hand, the best available experiments show good quantization of conductance, indicating that the backscattering remains negligible. This is most likely the result of smoothness of the confining potential. Indeed, the backscattering involves the change of the electron wavevector by $2 k_{F}$. Thus an external potential that is smooth at the scale of interparticle distance $n^{-1}$ will cause exponentially weak backscattering. In this paper we assume that the external potential is sufficiently smooth, so that the backscattering can be neglected.

Under the above conditions the low-energy properties of the system may be described by the bosonized Hamiltonian (2)-(4), but with position-dependent parameters $u_{\rho, \sigma}, K_{\rho, \sigma}, g_{1 \perp}$. In this paper we assume that the temperature is small compared with the bandwidth $D_{\rho} \sim \hbar n u_{\rho}$ of the Hamiltonian $H_{\rho}$, so that the discussion of the effect of the charge modes on the conductance presented in Sec. III is valid. On the other hand, we will be interested in the case of temperature comparable with the bandwidth $D_{\sigma} \sim J$ of the Hamiltonian $H_{\sigma}$. In this regime the bosonized version (4) of $H_{\sigma}$ is not applicable, and one should instead use the Heisenberg model (10).

Since the exchange constant (11) strongly depends on the electron density $n(x)$, the parameter $J$ in Eq. (10) should also be considered position-dependent. In particular, the strength of the exchange coupling between the two spins at the neighboring sites $l$ and $l+1$ of the Wigner lattice is a function of the coordinate $x_{l}$ of the $l$-th electron: $J=J\left(x_{l}\right)$. It is important to note that in the presence of electric current $I$ the Wigner lattice moves, so the coordinate $x_{l}$ of the $l$-th lattice site depends not only on $l$, but also on time.

The time dependence of $x_{l}$ can be accounted for by noting that if during the time interval $t$ a number $q(t)$ of electrons have moved from the left lead to the right one, the $l$-th site of the lattice has shifted to the $(l+q)$-th position. Thus the time dependence of the positions of the lattice sites can be accounted for by replacing $l \rightarrow$ $l+q(t)$, and the Hamiltonian $H_{\sigma}$ takes the form

$$
H_{\sigma}=\sum_{l} J[l+q(t)] \boldsymbol{S}_{l} \cdot \boldsymbol{S}_{l+1}
$$

Note that in this approximation the electric current $I=$ $e \dot{q}(t)$ is assumed to be uniform throughout the wire. This is true in the dc limit $\omega \ll u_{\rho} / L$.

It is important to note that the form (30) of the Hamiltonian $H_{\sigma}$ violates the spin-charge separation. Indeed, the coupling between the spins depends on the amount of charge that passed through the wire, which is related to the field $\phi_{\rho}$, see Eq. (24). As a result, the conductance of a quantum wire connected to bulk leads may be affected by the spin excitations. ${ }^{64}$

To find the effect of spin subsystem on the conductance, one could substitute the expression (24) for $q(t)$ into Eq. (30), and consider the complete Hamiltonian $H_{\rho}+H_{\sigma}$ without relying on spin-charge separation. In this approach one needs to add to the Hamiltonian a term describing the applied bias, and evaluate the electric current. However, it is more convenient to treat the current $I(t)$ in the wire as an external parameter. In this case $q(t)$ is also a parameter, and the Hamiltonians $H_{\rho}$ 
and $H_{\sigma}$ still commute. The only consequence of the violation of spin-charge separation in this approach is the dependence of $H_{\sigma}$ on the current $I(t)$.

The presence of an oscillating parameter (25) in the Hamiltonian (30) may lead to creation of spin excitations. Using the approach of Sec. III] we will calculate the energy dissipated into spin excitations in unit time. In the limit of weak current, the dissipation is found in the second order of the perturbation theory in the amplitude $I_{0}$ of current oscillations. Thus in addition to the plasmon result for the energy $W$ dissipated in unit time, we will obtain a similar contribution of the spin modes:

$$
W=\frac{1}{2} I_{0}^{2} R_{\rho}+\frac{1}{2} I_{0}^{2} R_{\sigma} .
$$

Comparing this result with the Joule heat law $W=$ $\frac{1}{2} I_{0}^{2} R$, we conclude that the resistance $R$ of the wire is given by sum of two independent contributions,

$$
R=R_{\rho}+R_{\sigma} .
$$

Note that the first term in this expression is already known, Eq. (29). The second term is discussed in Sec.

It is interesting to point out that the result (32) may be interpreted as a total resistance of the charge and spin subsystems connected in series, whereas naively one might expect a parallel connection. The reason is that the spins do not directly respond to the applied voltage, as required for the latter interpretation. Instead, the spin subsystem responds to the electric current. Thus the Hamiltonians $H_{\rho}$ and $H_{\sigma}$ become independent in the regime of applied current, in analogy with the problem of two independent resistors connected in series. A result similar to Eq (32) has been obtained for the resistivity of two-dimensional strongly interacting systems in Ref. 49.

\section{SPIN CONTRIBUTION TO THE RESISTANCE}

To find the contribution $R_{\sigma}$ of the spin subsystem to the resistance of the device, we study the dissipation of energy into spin excitations caused by the time dependence of the Hamiltonian (30). We start by performing the Jordan-Wigner transformation (13) and converting the Hamiltonian to the fermionic form

$$
\begin{aligned}
H_{\sigma}= & \frac{1}{2} \sum_{l} J[l+q(t)]\left[\left(a_{l}^{\dagger} a_{l+1}+a_{l+1}^{\dagger} a_{l}\right)\right. \\
& \left.+2\left(a_{l}^{\dagger} a_{l}+\frac{1}{2}\right)\left(a_{l+1}^{\dagger} a_{l+1}+\frac{1}{2}\right)\right] .
\end{aligned}
$$

In the absence of the external magnetic field the average $z$-component of the spin at every site of the lattice must vanish. Thus according to Eq. (13) the occupation of each site is $\left\langle a_{l}^{\dagger} a_{l}\right\rangle=\frac{1}{2}$. This means that the Fermi level is in the middle of the band, $\mu=0$.

The exchange $J[y]$ strongly depends on the position $y$. Inside the wire the electron density is low, $n a_{B} \ll 1$, and

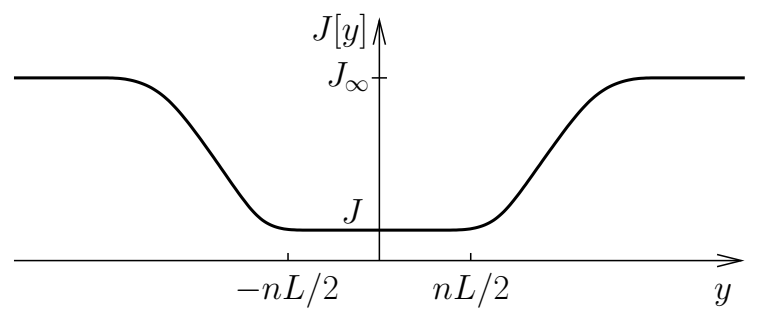

FIG. 1: Scketch of the dependence $J[y]$ in our model. Inside the wire, $|y|<n L / 2$, the exchange $J$ is exponentially small, Eq. (11). As one moves toward the leads, $J$ grows, and at $y \rightarrow \infty$ it saturates at $J_{\infty} \sim E_{F}$.

the exchange is exponentially small, Eq. (11). As the wire connects to the bulk leads, the density $n(x)$ begins to grow. At $n a_{B} \sim 1$ the exchange $J$ becomes of the order of the Fermi energy, see Eqs. (11), (12).

Strictly speaking, the Wigner crystal picture is valid only at $n a_{B} \ll 1$, that is as long as $J \ll E_{F}$. On the other hand, we will be interested in the properties of the system at low energies $D \ll E_{F}$. Thus at $J \sim E_{F}$ when the Wigner crystal picture fails, we are only concerned with the energy scales much lower than $J$. As we saw in Sec. III at those scales one can use the bosonized Hamiltonian (41) regardless of the applicability of the Wigner crystal description. Thus we can ignore the difference between the Wigner crystal and weakly interacting electron gas at large density $n \gg a_{B}$, and simply assume that in the leads the exchange $J$ saturates at $J_{\infty} \sim E_{F}$.

The properties of the function $J[y]$ can thus be summarized as follows,

$$
J[y]= \begin{cases}J \ll E_{F}, & \text { at }|y|<n L / 2, \\ J_{\infty} \sim E_{F}, & \text { at }|y| \rightarrow \pm \infty,\end{cases}
$$

see Fig. 1. Note that $y$ is the coordinate on the Wigner lattice. Since we consider the limit of very smooth confining potential, all the physical quantities change very little at the interparticle distance. We therefore assume that $J[y]$ is a slowly varying function: $|d J / d y| \ll J[y]$.

\section{A. XY model}

The Hamiltonian (33) describes a system of strongly interacting fermions. As a first approximation we will simplify the problem by neglecting the interactions between the fermions,

$$
H_{\sigma}^{X Y}=\frac{1}{2} \sum_{l} J[l+q(t)]\left(a_{l}^{\dagger} a_{l+1}+a_{l+1}^{\dagger} a_{l}\right)
$$

This Hamiltonian corresponds to the fermionized version of the XY model of a spin chain, in which the coupling of the $z$-components of spin operators is neglected. This approximation violates the $\mathrm{SU}(2)$ symmetry of the problem, and is therefore rather crude. On the other hand, 
the resistance $R_{\sigma}$ can be found exactly for model (35), and the result will provide considerable insight into the properties of model (33).

Hamiltonian (35) represents an inhomogeneous version of the the tight-binding model of lattice fermions. In the uniform case, $J[y]=$ const, the spectrum is well known,

$$
\epsilon(k)=J \sin k,
$$

where the wavevector $k$ is measured from $k_{F}=\pi / 2$. One can either assume that $k$ varies in the interval $-\pi<k<$ $\pi$, or choose $0<k<\pi$ and treat Eq. (36) as spectra of two branches of excitations, the particles and holes.

In the absence of electric current in the wire one can omit $q(t)$ in the Hamiltonian (35) and view it as a tightbinding model with slowly varying bandwidth $2 J[y]$. In the leads the bandwidth $2 J_{\infty}$ is very large; it narrows down to a very small value $2 J$ in the wire, Eq. (34). The particles moving toward the wire in one of the leads cross to the other lead if their energies are below the small exchange $J$ in the wire; the particles with $\epsilon>J$ are reflected.

In the presence of the electric current $I$, the constriction of the band in the Hamiltonian (35) moves with respect to the lattice with velocity $\dot{q}=I / e$. The particles reflected from the moving constriction change their energy. These processes lead to the dissipation of energy and contribute to the resistance $R_{\sigma}$.

For non-interacting fermions, the problem of evaluating the energy $W$ dissipated in unit time by a moving scatterer can be solved for arbitrary reflection coefficient $\mathcal{R}(\epsilon)$, see Appendix [C] Here we find $W$ in the semiclassical limit, which is valid for very slowly varying bandwidth $J[y]$, when $\mathcal{R}(\epsilon)=\theta(\epsilon-J)$.

In the limit of slowly varying $J[y]$ one can apply the result (36) for the spectrum of particles at every point in space, and treat the excitations as classical particles with energy

$$
H(y, p, t)=J[y+v t] \sin \frac{p}{\hbar} .
$$

Here $y$ is the coordinate of the particle, $p$ is its momentum, and $v=I / e$ is the velocity of the constriction. For simplicity we will consider the case of dc current, $I=$ const. To find the linear conductance of the quantum wire, one can limit oneself to the case of very small current, and assume $v \ll T / \hbar, J / \hbar$.

The time-dependent energy (37) should be treated as a Hamilton function, and the trajectory of the particle can, in principle, be found by solving the classical Hamilton equations. One can easily check that the quantity

$$
E(y, p, t)=H(y, p, t)+p v
$$

is an integral of motion. It has the meaning of energy of the particle in the frame moving at the speed of the constriction.

A particle with energy $\epsilon \sim T$ moving in the right direction has a very low momentum $p$ when it is in the leads, $p / \hbar=\epsilon / J_{\infty} \sim T / E_{F} \ll 1$. Thus its integral of motion $E(y, p, t)=\epsilon$. As the particle approaches the constriction, its momentum increases, so that $E$ retains its value despite the decrease of the bandwidth $J$. At small $v$ the maximum allowed value of $E$ in the wire is reached at $p=\pi \hbar / 2$ and equals $E_{\max }=J+\frac{\pi}{2} \hbar v$. Thus at $\epsilon<J+\frac{\pi}{2} \hbar v$ the right-moving particle moves from the left lead to the right one, and its energy $\epsilon$ remains unchanged. If the energy $\epsilon$ exceeds $J+\frac{\pi}{2} \hbar v$, the particle cannot enter the wire. When its momentum reaches $\pi \hbar / 2$ at a point to the left of the constriction, the particle is reflected. Deep in the left lead its momentum is very close to $\pi \hbar$. Due to the $p v$ term in integral of motion (38), its energy $H$ decreases to $\epsilon-\pi \hbar v$.

Similarly, since a left-moving particle in the right lead with energy $\epsilon$ has momentum very close to $\pi \hbar$, its integral of motion (38) is $E=\epsilon+\pi \hbar v$. The condition $E<E_{\max }$ for transmission through the constriction for such particles means $\epsilon<J-\frac{\pi}{2} \hbar v$. As the particle reaches the left lead, the momentum is again near $\pi \hbar$, i.e., conservation of $E$ results in conservation of energy $H=\epsilon$. On the other hand, particles with energies $\epsilon>J-\frac{\pi}{2} \hbar v$ are reflected back to the right lead, and their momentum on the right-moving branch is near $p=0$. Thus the energy of these particles increases from $\epsilon$ to $\epsilon+\pi \hbar v$.

To summarize, the particles in the leads with energies $\epsilon<J-\frac{\pi}{2} \hbar v$ cross the constriction region without change of energy. The particles with energies $\epsilon>J+\frac{\pi}{2} \hbar v$ are always reflected by the constriction. The ones in the left lead decrease their energy by $\pi \hbar v$, while the ones in the right lead increase their energy by the same amount, so that these contributions to the total energy of the system compensate each other. Finally, in the narrow range of energies $J-\frac{\pi}{2} \hbar v<\epsilon<J+\frac{\pi}{2} \hbar v$ the rightmovers go through the constriction without change of energy, whereas the left-movers reflect back to the right lead with energy gain $\pi \hbar v$.

The total current of left moving particles and holes in the narrow energy interval of width $\pi \hbar v$ near $\epsilon=J$ is given by $\delta \dot{N}=(2 / h)(\pi \hbar v) f(J)$, where $f(\epsilon)=1 /\left(e^{\epsilon / T}+\right.$ $1)$ is the Fermi function. Thus the total energy transferred to the spin excitations in unit time is

$$
W^{X Y}=\pi \hbar v^{2} f(J)=I^{2} \frac{\pi \hbar}{e^{2}} f(J) .
$$

Comparing this result with the Joule heat law $W=$ $I^{2} R$, we obtain the spin contribution to the resistance

$$
R_{\sigma}^{X Y}=\frac{h}{2 e^{2}} f(J)
$$

At low temperature $T \ll J$ most of the particles have energies below $J$ and pass through the constriction elastically. Only an exponentially small fraction of particles are reflected and contribute to the dissipation. Thus the result (40) is exponentially small at low temperatures, $R_{\sigma}^{X Y} \simeq\left(h / 2 e^{2}\right) e^{-J / T}$. As the temperature is increased, a greater fraction of the particles are reflected by the constriction, and the $R_{\sigma}^{X Y}$ increases. In the limit $T / J \rightarrow \infty$ 
all the particles are reflected, and the resistance saturates at $R_{\sigma}^{X Y}=h / 4 e^{2}$.

In this section we studied the simplified XY model, in which the $z$-component of coupling in the Hamiltonian (30) was neglected. Thus the result (40) cannot be applied directly to the problem of conductance of a quantum wire in the Wigner crystal regime. However, much of the physics leading to Eq. (40) can be carried over to the case of the isotropic model (30).

\section{B. Isotropic coupling}

The problem of the isotropic inhomogeneous Heisenberg spin chain (30) is far more complicated than that of the XY model (35). However, it can still be somewhat simplified by assuming that $J[y]$ is a very slowly varying function. Then each moderately long section of the spin chain can be approximated by the homogeneous Heisenberg model. The latter allows for exact solution ${ }^{50}$ by means of Bethe ansatz. The low-energy excitations of the isotropic Heisenberg spin chain are spinons with energy spectrum 37.38

$$
\epsilon(k)=\frac{\pi J}{2} \sin k
$$

Although the spinons do not obey Fermi statistics, the similarity between Eq. (41) and the spectrum (36) of the excitations of XY model enables us to find the temperature dependence of $R_{\sigma}$ at $T \ll J$.

Indeed, most of the discussion leading to Eq. (40) did not rely on Fermi statistics of the excitations. One can apply the arguments of Sec. $\mathrm{VA}$ to the problem of scattering of spinons by the constriction of the band in the wire. In particular, one concludes that spinons with energies below $\pi J / 2$ pass through the constriction without scattering and do not change their energy. Thus the dissipation is exponentially small at $T \ll J$, and one finds $R_{\sigma} \propto \exp (-\pi J / 2 T)$

Since the occupation of states with high energy $\epsilon=$ $\pi J / 2$ at low temperature is exponentially small and independent of statistics, on can naively expect the resistance $R_{\sigma}$ to be given by the low-temperature asymptotics of Eq. (40) upon replacement $J \rightarrow \pi J / 2$. Then one obtains

$$
R_{\sigma}=R_{0} \exp \left(-\frac{\pi J}{2 T}\right)
$$

This approach gives the prefactor $R_{0}=h / 2 e^{2}$.

Unfortunately the analogy between spinons and fermion excitations of the XY model does not enable one to find the prefactor in Eq. (42). Unlike the excitations of the XY model, the spinons interact with each other, and the energy of a spinon is affected by the presence of other spinons. In the limit $T \rightarrow 0$ the density of other spinons is small, and the energy is given by Eq. (41). At finite $T$ the result (41) may acquire a small correction.
The exponent of Eq. (42) is determined by the maximum energy of a spinon $\pi J / 2$. Even a small correction to this energy may affect the prefactor $R_{0}$.

At high temperature $T \gg J$ the resistance contribution $R_{\sigma}$ evaluated within the XY model approximation saturates, because in this regime all the excitations are reflected by the constriction. This feature is preserved in the model with isotropic coupling, as at $J \rightarrow 0$ the spin excitations cannot propagate through the wire. The saturation value of $R_{\sigma}$ at high temperatures can be found by noticing that in the part of the system away from the constriction, where $J[l] \simeq J_{\infty} \gg T$, one can still bosonize the Hamiltonian (33) and use the form (4). Inside the constriction the bosonization is not applicable, and this region is modeled by imposing a boundary condition on the bosonic field $\phi_{\sigma}$ corresponding to the fact that there is no spin propagation between the regions of large positive and negative $l$.

The form of the boundary condition can be deduced by replacing the constriction (34) of the bandwidth $D_{\sigma} \sim$ $J[l]$ in the Hamiltonian (33) with a high potential barrier for the fermions. The barrier is modeled by a large backscattering term $v\left(a_{L}^{\dagger} a_{R}+a_{R}^{\dagger} a_{L}\right)$ at site $l=-q(t)$. Upon the bosonization transformation (15) this term becomes $-\left.\tilde{v} \cos \left[\sqrt{2} \phi_{\sigma}(y)-2 k_{F} y\right]\right|_{y=-q(t)}$, with $k_{F}$ on the lattice being $\pi / 2$. Since this scattering term is very strong, $\tilde{v} \rightarrow \infty$, it pins the field $\phi_{\sigma}(-q(t), t)$ to the value $-\sqrt{2} k_{F} q(t)=-(\pi / \sqrt{2}) q(t)$. This time-dependent boundary condition leads to the emission of spin waves, in analogy with Sec.IIIA where the boundary condition (24) gave rise to plasmon waves (26). In the limit of weak current, $I \sim e \omega q \rightarrow 0$, the wavelength of the spin waves $\sim J_{\infty} / \hbar \omega$ is much larger than $q(t)$, and instead of imposing the boundary condition at $y=-q(t)$ one can impose it at $y=0$. Then the boundary condition becomes

$$
\phi_{\sigma}(0, t)=-\frac{\pi}{\sqrt{2}} q(t)
$$

Note that up to inessential negative sign Eq. (43) is identical to the boundary condition (24). The respective Hamiltonians (3) and (4) are also essentially identical at low energies, as the sine-Gordon term is irrelevant. One can therefore carry over the results of Sec. IIIA for the dissipation of energy into plasmon waves and the resulting contribution to the resistance. Adapting Eq. (28) to the parameters of Hamiltonian (4), we find

$$
R_{\sigma}=\frac{h}{2 K_{\sigma} e^{2}} .
$$

In the dc limit the frequency of the driving force $\omega \rightarrow 0$, and the wavelength of the spin waves is very long. Thus the parameter $K_{\sigma}$ in Eq. (44) is taken at large distances from the constriction, where the SU(2) symmetry demands $K_{\sigma}=1$. Consequently the spin contribution to the resistance in the model with isotropic coupling is given by

$$
R_{\sigma}=\frac{h}{2 e^{2}}
$$


On the other hand, the XY model (35) does not possess the $\mathrm{SU}(2)$ symmetry, and the bosonization procedure (15) gives the quadratic part of Hamiltonian (4) with $K_{\sigma}=2$. Then Eq. (44) predicts $R_{\sigma}^{X Y}=h / 4 e^{2}$, in agreement with $T \gg J$ asymptotics of Eq. (40).

\section{DISCUSSION OF THE RESULTS}

The quantity most commonly measured in experiments with quantum wire devices is the linear conductance. In our theory its value is given by

$$
G=\frac{1}{R_{\rho}+R_{\sigma}},
$$

c.f. Eq. (32). The contributions $R_{\rho}$ and $R_{\sigma}$ to the resistance of the wire are determined by the properties of the charge and spin excitations of the system, respectively.

Throughout this paper we consider the case of relatively low temperature, $T \ll D_{\rho} \sim \hbar n u_{\rho}$. In this regime the contribution of the charge modes is well known: $R_{\rho}=h / 2 e^{2}$ (see also Sec. IIIB). Raising the temperature above $D_{\rho}$ leads to thermal smearing of conductance plateaus. No interesting electron correlation effects are expected in this case.

At not too low electron density $n \gtrsim a_{B}^{-1}$ the bandwidth $D_{\rho} \sim D_{\sigma} \sim E_{F}$ is the only relevant energy scale of the problem. Then at $T \ll E_{F}$ the contribution $R_{\sigma}$ vanishes, and the conductance takes the well-known quantized value $G=2 e^{2} / h$. On the other hand, in the interesting case of low density $n \ll a_{B}^{-1}$ another energy scale, the exchange constant $J$, appears in the problem. This scale is exponentially small, Eq. (11); in particular, $J \ll D_{\rho}$. In the limit of low temperature $T \rightarrow 0$ the contribution $R_{\sigma}$ still vanishes. More specifically, at $T \ll J$ we predict activated temperature dependence (42) of $R_{\sigma}$, with activation temperature $\pi J / 2$. At higher temperatures $R_{\sigma}$ grows, and at $T \gg J$ it saturates at the universal value $R_{\sigma}=h / 2 e^{2}$, see Eq. (45). Combining these results with Eq. (46), we obtain

$$
G=\frac{e^{2}}{h}, \quad J \ll T \ll D_{\rho} .
$$

This is our main result. It corresponds to an additional quantized plateau of conductance of a quantum wire at low electron density. The value of the conductance at this plateau is exactly one half of the quantized conductance $2 e^{2} / h$.

The plateaus of conductance at $e^{2} / h$ have been observed at low electron densities in several experiments 10.11 .12 .13 The authors of Refs. 10 11.12 13 attributed this feature to the spontaneous spin polarization in quantum wires. This interpretation contradicts to the theorem by Lieb and Mattis, ${ }^{51}$ stating that the ground state of a $1 \mathrm{D}$ electron system cannot be spin-polarized in the absence of magnetic field. One can hypothesize that the ferromagnetism in quantum wires is possible because the electrons are not truly one-dimensional; however to the best of our knowledge, no such theory is available at this time. In our theory the spin structure of the Wigner crystal state is described by the Heisenberg model (10) with positive exchange constant $J$, corresponding to antiferromagnetic coupling. Thus the ground state of the Wigner crystal in not spin-polarized, in agreement with the theorem 51

The temperature dependence of the conductance of a quantum wire device obtained in this paper is similar to the behavior observed in experiments on 0.7 structure in quantum point contacts 5.6 .7 .8 .9 In agreement with experiments, conductance (46) remains $2 e^{2} / h$ at $T \rightarrow 0$, but develops a negative correction at finite temperature. The activated temperature dependence of the correction following from Eq. (42) is consistent with the measurements of Ref. 9. At high temperature the correction saturates, and the conductance develops a new plateau. Contrary to the experiments, $, 6,7$ this plateau is at one half of the quantized value $2 e^{2} / h$, rather than at $0.7 \times\left(2 e^{2} / h\right)$. The relation between the plateau at $e^{2} / h$ and the 0.7 structure was studied experimentally in Ref. 12. It was found that the quasi-plateau at $0.7 \times\left(2 e^{2} / h\right)$ is observed in short wires, whereas in longer wires it shifts toward $e^{2} / h$. In this paper we assume that the wire is long, so that the parameters of the system, such as the confining potential, Fermi energy, and exchange constant $J$, do not change significantly at the interparticle distance. It would be interesting to generalize our approach to the case of shorter wires and see whether the physics discussed in this paper may be responsible for the 0.7 structure in quantum point contacts.

To test the relevance of our theory to the experiments $\frac{10,11,12,13}{3}$ showing plateaus at $e^{2} / h$, one can check whether the experimental temperature exceeds the exchange energy $J$. Due to the strong exponential dependence (11) of $J$ on the density, the uncertainly of $n$ may make the estimation of $J$ difficult. Instead one may be able to determine $J$ experimentally by applying magnetic field. Indeed, if the magnetic field $B$ exceeds a certain critical value $B_{c} \propto J$, the spin chain becomes completely spin polarized. The magnitude of the critical field $B_{c}$ can be found by considering the spin-polarized state in a strong field $B$ with a single spin-flip excitation. The energy of such an excitation $|g| \mu_{B} B$ is reduced by $2 J$ due to coupling to neighboring spins. (Here $g$ is the Lande factor, and $\mu_{B}$ is Bohr magneton.) Thus the complete polarization occurs at $B>B_{c}$, where

$$
B_{c}=\frac{2 J}{|g| \mu_{B}} \text {. }
$$

By measuring the critical field $B_{c}$ required to achieve complete polarization of the spin chain one can determine the exchange constant $J$.

In the case of non-interacting electrons at zero temperature the conductance does not depend on the magnetic field and remains $2 e^{2} / h$ until the electron gas becomes completely spin polarized at $B>B_{c}^{(0)}=E_{F} / 4|g| \mu_{B}$. 
In a polarizing field only one spin channel is allowed in the wire, and the conductance reduces to $e^{2} / h$. In the case of a quantum wire at low electron density this behavior is preserved, but the step in conductance occurs at a much lower critical field (48). Indeed, although in the presence of magnetic field spinons are no longer the elementary excitations of a spin chain, at $B<B_{c}$ one can introduce modified elementary excitations with similar properties 52 Then by repeating the arguments of Sec. $\mathrm{VB}$ one concludes that the low energy excitations present in the system at $T \rightarrow 0$ cross the wire elastically, resulting in $R_{\sigma}=0$ and total conductance $G=2 e^{2} / h$. At $B>B_{c}$ the wire is completely spin polarized, and the spin excitations in the leads are reflected by the wire. This situation is completely analogous to the case of high temperature considered in Sec. VB In particular, the resistance $R_{\sigma}$ can be found by bosonizing the electron system in the leads and imposing the boundary condition (43) on the field $\phi_{\sigma}$. This again leads to $R_{\sigma}=h / 2 e^{2}$ and reduces the conductance of the device to $e^{2} / h$. Thus one can find the critical field (48) and the exchange constant $J$ by measuring the magnetic field at which the conductance drops from $2 e^{2} / h$ to $e^{2} / h$.

Apart from the experiments with GaAs quantum wires, quantization of conductance at $G=e^{2} / h$ in the absence of magnetic has been observed in carbon nanotubes. 53 This anomalous quantization occurs when the current is forced to flow through the narrow tip of the tube. At small radius of the nanotube the Coulomb interactions between electrons become effectively stronger, and could conceivably suppress the exchange coupling $J$ of the electron spins below the temperature. Our result (47) would then explain the experimental data 53

\section{Acknowledgments}

The author acknowledges helpful discussions with A. V. Andreev, A. M. Finkel'stein, L. I. Glazman, A. I. Larkin, R. de Picciotto, M. Pustilnik, and P. B. Wiegmann and the hospitality of Bell Laboratories, where part of this work was carried out. This work was supported by the U.S. DOE, Office of Science, under Contract No. W-31-109-ENG-38, by the Packard Foundation and by NSF Grant DMR-0214149.

\section{APPENDIX A: ESTIMATE OF THE EXCHANGE CONSTANT $J$}

Here we estimate the exchange constant in an infinite 1D Wigner crystal with the lattice constant $b=1 / n$. Following the idea of Häusler ${ }^{36}$ we evaluate $J$ for two spins at neighboring sites $l=0$ and $l=1$ using an approximation where the only dynamical variable is the distance $x=x_{1}-x_{0}$ between the two electrons. In this approximation, $x_{0}+x_{1}=b$ and all the other electrons $(l \neq 0,1)$ are at fixed positions $x_{l}=l b$. Then the Coulomb poten- tial takes the form

$$
U(x)=\frac{e^{2}}{\varepsilon|x|}+\sum_{l \neq 0,1}\left(\frac{e^{2}}{\varepsilon\left|\frac{b-x}{2}-l b\right|}+\frac{e^{2}}{\varepsilon\left|\frac{b+x}{2}-l b\right|}\right) .
$$

This potential has two degenerate minima at $x= \pm b$ corresponding to $x_{0}=0, x_{1}=b$ and $x_{0}=b, x_{1}=0$. In the limit of strong Coulomb potential the tunneling between these two states gives rise to exponentially small splitting $\Delta E$ of the doublet.

The ground state wavefunction of this system is an even function of $x$, and is therefore symmetric with respect to permutation $x_{0} \leftrightarrow x_{1}$, while the first excited state is antisymmetric. The two states correspond to the values of the total spin of the two-electron system $S=0$ and $S=1$, respectively. Thus the energy of the two components of the doublet can be written in terms of the electron spin operators at the two sites as $E_{0}+J \boldsymbol{S}_{0} \cdot \boldsymbol{S}_{1}$, where $J$ is identified with the level splitting $\Delta E$.

Strictly speaking the infinite series in Eq. A1 diverges. This is due the long range nature of the Coulomb interactions. In practice the interactions are screened at large distances by remote gates. Instead of modifying the Coulomb potential to account for the gate, it will be more convenient to simply subtract from Eq. (A1) a divergent constant $U(b)$. Then the series converges, and in the important region $|x|<3 b$ the potential can be presented in analytic form as

$$
\begin{aligned}
& U(x)=\frac{e^{2}}{\varepsilon b}[F(x / b)-F(1)] \\
& F(z)=\frac{1}{|z|}-2 \psi\left(\frac{3-z}{2}\right)-2 \psi\left(\frac{3+z}{2}\right),
\end{aligned}
$$

where $\psi(z)$ is the digamma function.

Evaluation of the energy level splitting $\Delta E$ for a particle of mass $m$ in a double-well potential $U(x)$ is a wellknown problem of quantum mechanics,, 54 and the result 65 is given by

$$
\Delta E=\frac{\hbar \omega}{\sqrt{e \pi}} \exp \left(-\frac{1}{\hbar} \int_{-a}^{a} \sqrt{2 m[U(x)-\hbar \omega / 2]} d x\right) .
$$

Here $\omega=\sqrt{U^{\prime \prime}(b) / m}$ is the frequency of small oscillations near the minima $x= \pm b$ of the potential $U(x)$, and $x= \pm a$ are the classical turning points at energy $\hbar \omega / 2$, i.e., $a=b-\sqrt{\hbar / m \omega}$.

To evaluate $\Delta E$ with the correct prefactor, one has to carefully account for the small energy $\hbar \omega / 2$ in the exponential. The resulting level splitting can be written as

$$
\Delta E=\frac{2}{\sqrt{\pi}} \sqrt{\hbar \omega^{3} m b^{2}} e^{\xi} e^{-S_{0}}
$$

where

$$
\begin{aligned}
S_{0} & =\frac{1}{\hbar} \int_{-b}^{b} \sqrt{2 m U(x)} d x \\
\xi & =\int_{0}^{1}\left(\sqrt{\frac{U^{\prime \prime}(b) b^{2}}{2 U(b z)}}-\frac{1}{1-z}\right) d z .
\end{aligned}
$$


An alternative solution ${ }^{55}$ of the problem using the instanton technique leads to a result that can be also presented in the form A5 - A7 .

In order to apply this result to the evaluation of the exchange constant $J$, one has to keep in mind that $x$ is the relative position of two neighboring electrons, $x=$ $x_{0}-x_{1}$, and replace the mass in Eqs. (A5), (A6) with the reduced mass $m / 2$. One then finds $S_{0}=\eta \sqrt{b / a_{B}}$ with the numerical coefficient

$$
\eta=\int_{-1}^{1} \sqrt{F(z)-F(1)} d z \approx 2.817 .
$$

Substitution of this result into Eq. A5 gives the leading exponential behavior of Eq. (11).

The numerical parameter $\xi$ defined by Eq. (A7) depends only on the shape of the barrier separating the two minima of potential $U(x)$. For the potential A2, we find

$$
\xi=\int_{0}^{1}\left(\sqrt{\frac{F^{\prime \prime}(1)}{2[F(z)-F(1)]}}-\frac{1}{1-z}\right) d z \approx-0.423 .
$$

Substituting this result in Eq. A5 we find the exchange constant

$$
J=\frac{\kappa \hbar^{2}}{m \sqrt[4]{b^{5} a_{B}^{3}}} \exp \left(-\eta \sqrt{\frac{b}{a_{B}}}\right),
$$

with $\kappa \approx 2.203$. Expressing the prefactor in terms of the Fermi energy, we obtain Eq. (12).

It is worth mentioning that because of the singularity of the potential $U(x)$ at $x=0$, the validity of the WKB approximation used in the derivation 54 of formula (A4 is limited to $|x| \gg a_{B}$. Moreover, since the potential (A2) is not integrable up to the singularity, it represents an impenetrable barrier ${ }^{58}$ Thus the true value of the level splitting for potential (A2) is $\Delta E=0$. On the other hand, the electrons in a quantum wire are not strictly one-dimensional due to the finite width $w$ of the wire. As a result the singularity of the Coulomb interaction potential is cut off at short distances $x \sim w$. In GaAs devices $w \gtrsim a_{B}$, which justifies the WKB approximation. In carbon nanotubes it may possible to achieve the regime $w \ll a_{B}$; a more sophisticated approach to the calculation of the exchange constant $J$ is required in this case. 59

\section{APPENDIX B: RESISTANCE OF A QUANTUM WIRE}

Let us derive the resistance (28) of an infinite quantum wire in the regime of applied current. The wire is modeled by the Hamiltonian (3) with the time-dependent boundary condition (24). It is convenient to apply to the Hamiltonian a unitary transformation

$$
U=\exp \left(-i \frac{\pi q(t)}{\sqrt{2}} \int_{-\infty}^{\infty} \Pi_{\rho}(x) d x\right)
$$

which shifts the charge field $\phi_{\rho}(x) \rightarrow \phi_{\rho}(x)+\frac{\pi}{\sqrt{2}} q(t)$. As a result the boundary condition (24) is replaced with $\phi_{\rho}(0, t)=0$, but the Hamiltonian (3) acquires a timedependent perturbation

$$
V=-i \hbar U^{\dagger} \partial_{t} U=-\frac{\pi \hbar}{\sqrt{2}} \dot{q}(t) \int_{-\infty}^{\infty} \Pi_{\rho}(x) d x .
$$

The perturbation (B2) leads to excitation of plasmons and to dissipation of energy into the Luttinger liquid. To find the energy $W$ dissipated in unit time, it is convenient to diagonalize $H_{\rho}$ by introducing the plasmon destruction operators

$$
b_{k}=\int \theta(k x) \sin k x\left(\frac{1}{\pi} \sqrt{\frac{|k|}{K_{\rho}}} \phi_{\rho}(x)+i \sqrt{\frac{K_{\rho}}{|k|}} \Pi_{\rho}(x)\right) d x \text {, }
$$

where $\theta(y)$ is the unit step function. Note that in order to satisfy the boundary condition $\phi_{\rho}(0, t)=0$ the wavefunctions of the plasmons were chosen in the form $\varphi_{k}(x)=\sqrt{2 / \pi} \theta(k x) \sin k x$; positive and negative $k$ correspond to excitations to the right and left of the boundary $x=0$, respectively.

Upon the transformation to the new variables (B3), the two terms in the Hamiltonian take the form

$H_{\rho}=\int_{-\infty}^{\infty} \hbar \omega_{k} b_{k}^{\dagger} b_{k} d k, \quad V=\frac{i \hbar I_{0} \cos \omega t}{e \sqrt{2 K_{\rho}}} \int_{-\infty}^{\infty} \frac{b_{k}-b_{k}^{\dagger}}{\sqrt{|k|}} d k$

The perturbation $V$ leads to both emission and absorption of plasmons with energy $\hbar \omega$. The total energy dissipated in unit time can be evaluated using the Fermi golden rule as

$$
W=\frac{2 \pi}{\hbar}\left(\frac{\hbar I_{0}}{2 e \sqrt{2 K_{\rho}}}\right)^{2} \frac{2}{\hbar \omega}\left[\left(1+f_{k}\right) \hbar \omega-f_{k} \hbar \omega\right] .
$$

Regardless of the values of the plasmon occupation numbers $f_{k}$, expression (B5) reduces to $W=\frac{1}{2} I_{0}^{2} R_{\rho}$ with the resistance (28).

\section{APPENDIX C: DISSIPATION OF ENERGY BY A SCATTERER IN A FERMI GAS}

Let us consider the dissipation of energy in a noninteracting Fermi gas in the presence of a moving scatterer. We assume that the single-particle Hamiltonian has the general form

$$
H(y, p, t)=H_{0}\left(y+q_{0} \sin \omega t, p\right) .
$$

Here the hermitian operator $H_{0}(y, p)$ is independent of the coordinate $y$ in the regions corresponding to the leads, $y \rightarrow \pm \infty$. The Hamiltonian (35) obviously satisfies these conditions for smoothly varying $J[y]$ after the 
discrete site number $l$ is replaced by a continuous coordinate $y$. The $y$-dependent central part of the Hamiltonian $H_{0}(y, p)$ can be viewed as a scatterer with energydependent reflection coefficient $\mathcal{R}(\epsilon)$. Condition (C1) implies that the position of the scatterer oscillates with amplitude $q_{0}$.

In the limit of small $q_{0}$ one can expand Eq. (C1) and present the Hamiltonian as

$$
H(y, p, t)=H_{0}(y, p)+q_{0} \sin \omega t \partial_{y} H_{0}(y, p) .
$$

The time-dependent perturbation leads to absorption and emission of energy quanta $\hbar \omega$ by the fermions. The rates of these processes may be found using the Fermi golden rule, and one obtains the increase $W$ of the energy of the system in unit time in the form

$$
\begin{aligned}
W= & \frac{2 \pi}{\hbar} \hbar \omega \iint d k d k^{\prime}\left|\frac{q_{0}}{2}\left[\partial_{y} H_{0}\right]_{k k^{\prime}}\right|^{2} \\
& \times\left[f\left(\epsilon_{k}\right)-f\left(\epsilon_{k}^{\prime}\right)\right] \delta\left(\epsilon_{k}-\epsilon_{k^{\prime}}+\hbar \omega\right) .
\end{aligned}
$$

Here $k$ labels the eigenstates of Hamiltonian $H_{0}$ with energies $\epsilon_{k}$. The occupation numbers of these states are given by the Fermi function $f\left(\epsilon_{k}\right)$. The eigenfunctions have scattering wave asymptotics

$$
\psi_{k}(y)=\frac{1}{\sqrt{2 \pi}} \times \begin{cases}e^{i k y}+r_{k} e^{-i k y}, & \text { at } y \rightarrow-\infty, \\ t_{k} e^{i k y}, & \text { at } y \rightarrow+\infty .\end{cases}
$$

for positive $k$ and

$$
\psi_{k}(y)=\frac{1}{\sqrt{2 \pi}} \times \begin{cases}t_{k} e^{i k y}, & \text { at } y \rightarrow-\infty, \\ e^{i k y}+r_{k} e^{-i k y}, & \text { at } y \rightarrow+\infty\end{cases}
$$

for negative $k$. Here $r_{k}$ and $t_{k}$ are the reflection and transmission amplitudes; the reflection coefficient is defined as $\mathcal{R}\left(\epsilon_{k}\right)=\left|r_{k}\right|^{2}$.

In the limit of low frequency $\omega \rightarrow 0$ expression (C3) can be further simplified,

$$
W=\frac{\pi\left(\omega q_{0}\right)^{2}}{\hbar v_{F}^{2}} \int d \epsilon_{k}\left[-f^{\prime}\left(\epsilon_{k}\right)\right]\left[\zeta_{+}\left(\epsilon_{k}\right)+\zeta_{-}\left(\epsilon_{k}\right)\right]
$$

Here we have approximated the energies near the Fermi level as $\epsilon_{k}=\hbar v_{F}\left(|k|-k_{F}\right)$, accounted for the double degeneracy of the energy levels $\epsilon_{k}$, and introduced

$$
\zeta_{ \pm}\left(\epsilon_{k}\right)=\lim _{k^{\prime} \rightarrow \pm k}\left|\left[\partial_{y} H_{0}\right]_{k k^{\prime}}\right|^{2}
$$

The matrix element $\left[\partial_{y} H_{0}\right]_{k, k^{\prime}}$ is defined as

$$
\left[\partial_{y} H_{0}\right]_{k k^{\prime}}=\int d y \psi_{k^{\prime}}^{*}(y)\left[\partial_{y} H_{0}\right] \psi_{k}(y)
$$

Integrating by parts and taking advantage of the fact that $\psi_{k}$ is an eigenfunction of $H_{0}$, we find

$$
\left[\partial_{y} H_{0}\right]_{k k^{\prime}}=\left(\epsilon_{k}-\epsilon_{k^{\prime}}\right) \int d y \psi_{k^{\prime}}^{*}(y) \partial_{y} \psi_{k}(y)
$$

To evaluate $\zeta_{ \pm}\left(\epsilon_{k}\right)$ we need to find the divergent at $k^{\prime} \rightarrow$ $\pm k$ part of the integral in Eq. (C9). Since the divergences originate at $y \rightarrow \pm \infty$, one can use the scattering wave asymptotics (C4) and (C5) in Eq. (C9). This results in

$$
\begin{aligned}
\zeta_{+}\left(\epsilon_{k}\right) & =\frac{1}{\pi^{2}}\left(\hbar v_{F} k_{F}\right)^{2}\left[\mathcal{R}\left(\epsilon_{k}\right)\right]^{2} \\
\zeta_{-}\left(\epsilon_{k}\right) & =\frac{1}{\pi^{2}}\left(\hbar v_{F} k_{F}\right)^{2} \mathcal{R}\left(\epsilon_{k}\right)\left[1-\mathcal{R}\left(\epsilon_{k}\right)\right]
\end{aligned}
$$

Substituting these results into Eq. (C6), we find

$$
W=\frac{\hbar}{\pi}\left(\omega q_{0}\right)^{2} k_{F}^{2} \int d \epsilon\left[-f^{\prime}(\epsilon)\right] \mathcal{R}(\epsilon)
$$

To apply this result to the evaluation of the spin contribution to the resistance within the XY model approximation, one should substitute $\omega q_{0}=I_{0} / e$ and $k_{F}=\pi / 2$. Then Eq. (C12) takes the form

$$
W=\frac{1}{2} I_{0}^{2} R_{\sigma}^{X Y}, \quad R_{\sigma}^{X Y}=\frac{h}{4 e^{2}} \int d \epsilon\left[-f^{\prime}(\epsilon)\right] \mathcal{R}(\epsilon) .
$$

The result for the resistance coincides with Eq. (40) for the appropriate reflection coefficient $\mathcal{R}(\epsilon)=\theta(|\epsilon|-J)$.
1 B. J. van Wees, H. van Houten, C. W. J. Beenakker, J. G. Williamson, L. P. Kouwenhoven, D. van der Marel, and C. T. Foxon, Phys. Rev. Lett. 60, 848 (1988).

2 D. A. Wharam, T. J. Thornton, R. Newbury, M. Pepper, H. Ahmed, J. E. F. Frost, D. G. Hasko, D. C. Peacock, D. A. Ritchie, and G. A. C. Jones, J. Phys. C 21, L209 (1988).

3 S. Tarucha, T. Honda, and T. Saku, Solid State Comm. 94, 413 (1995).

4 A. Yacoby, H. L. Stormer, N. S. Wingreen, L. N. Pfeiffer, K. W. Baldwin, and K. W. West, Phys. Rev. Lett. 77, 4612 (1996).

${ }^{5}$ K. J. Thomas, J. T. Nicholls, M. Y. Simmons, M. Pepper, D. R. Mace and D. A. Ritchie, Phys. Rev. Lett. 77, 135
(1996).

${ }^{6}$ K. J. Thomas, J. T. Nicholls, N. J. Appleyard, M. Y. Simmons, M. Pepper, D. R. Mace, W. R. Tribe, and D. A. Ritchie, Phys. Rev. B 58, 4846 (1998).

7 S. M. Cronenwett, H. J. Lynch, D. Goldhaber-Gordon, L. P. Kouwenhoven, C. M. Marcus, K. Hirose, N. S. Wingreen, and V. Umansky, Phys. Rev. Lett. 88, 226805 (2002).

8 A. Kristensen, J. Bo Jensen, M. Zaffalon, C. B. Sørensen, P. E. Lindelof, M. Michel, and A. Forchel, J. Appl. Phys. 83, 607 (1998).

9 A. Kristensen, H. Bruus, A. E. Hansen, J. B. Jensen, P. E. Lindelof, C. J. Marckmann, J. Nygard, C. B. Sørensen, F. Beuscher, A. Forchel, and M. Michel, Phys. Rev. B 62, 
10950 (2000)

10 B. E. Kane, G. R. Facer, A. S. Dzurak, N. E. Lumpkin, and R. G. Clark, L. N. Pfeiffer and K. W. West, Appl. Phys. Lett. 72, 3506 (1998).

11 K. J. Thomas, J. T. Nicholls, M. Pepper, W. R. Tribe, M. Y. Simmons, and D. A. Ritchie, Phys. Rev. B 61, R13365 (2000).

12 D. J. Reilly, G. R. Facer, A. S. Dzurak, B. E. Kane, R. G. Clark, P. J. Stiles, R. G. Clark, A. R. Hamilton, J. L. O'Brien, N. E. Lumpkin, L. N. Pfeiffer and K. W. West, Phys. Rev. B 63, 121311(R) (2001).

13 D. J. Reilly, T. M. Buehler, J. L. O'Brien, A. R. Hamilton, A. S. Dzurak, R. G. Clark, B. E. Kane, L. N. Pfeiffer, and K. W. West, Phys. Rev. Lett. 89, 246801 (2002).

14 C.-K. Wang and K.-F. Berggren, Phys. Rev. B 57, 4552 (1998).

15 H. Bruus and K. Flensberg, Semicond. Sci. Technol. 13, A30 (1998).

16 S. M. Reimann, M. Koskinen, and M. Manninen, Phys. Rev. B 59, 1613 (1999).

17 B. Spivak and F. Zhou, Phys. Rev. B 61, 16730, (2000).

18 V. V. Flambaum and M. Yu. Kuchiev, Phys. Rev. B 61, R7869 (2000).

19 T. Rejec, A. Rams̆ak, and J. H. Jefferson, Phys. Rev. B 62, 12985 (2000).

${ }^{20}$ H. Bruus, V. V. Cheianov, and K. Flensberg, Physica E 10, 97 (2001).

21 K. Hirose, S. S. Li, and N. S. Wingreen, Phys. Rev. B 63, 033315 (2001).

22 O. P. Sushkov, Phys. Rev. B 64, 155319 (2001); Phys. Rev. B 67, 195318 (2003).

${ }^{23}$ Y. Tokura and A. Khaetskii, Physica E, 12, 711 (2002).

${ }^{24}$ Y. Meir, K. Hirose, and N. S. Wingreen, Phys. Rev. Lett. 89, 196802 (2002).

25 G. Seelig and K. A. Matveev, Phys. Rev. Lett. 90, 176804 (2003).

26 A. A. Starikov, I. I. Yakimenko, and K.-F. Berggren, Phys. Rev. B 67, 235319 (2003).

27 E. Wigner, Phys. Rev. 46, 1002 (1934); Trans. Faraday Soc. 34, 678 (1938).

${ }^{28}$ F. D. M. Haldane, J. Phys. C 14, 2585 (1981).

${ }^{29}$ K. A. Matveev, Phys. Rev. Lett. 92, 106801 (2004).

30 I. E. Dzyaloshinskii and A. I. Larkin, Sov. Phys.-JETP 38, 202 (1974).

31 C. F. Coll III, Phys. Rev. B 9, 2150 (1974).

${ }^{32}$ H. J. Schulz, G. Cuniberti, and P. Pieri, in Field Theories for Low-Dimensional Condensed Matter Systems, ed. by G. Morandi et al. (Springer-Verlag, New York, 2000).

33 T. Giamarchi, Quantum Physics in One Dimension, (Clarendon Press, Oxford, 2004).

34 L.I. Glazman, I. M. Ruzin, and B. I. Shklovskii, Phys. Rev. B 45, 8454 (1992).

35 See, e.g., J.H. Jefferson and W. Häusler, Phys. Rev. B 54, 4936 (1996)

36 W. Häusler, Z. Phys. B 99, 551 (1996).

37 J. des Cloizeaux and J. J. Pearson, Phys. Rev. 128, 2131 (1962)

38 L. D. Faddeev and L. A. Takhtajan, Phys. Lett. 85A, 375
(1981).

39 V. V. Cheianov and M. B. Zvonarev, J. Phys. A 37, 2261 (2004); V. V. Cheianov and M. B. Zvonarev, cond-mat/0308470

40 G. A. Fiete and L. Balents, cond-mat/0403744

41 M. Ogata and H. Shiba, Phys. Rev. B 41, 2326 (1990).

${ }^{42}$ H. Shiba and M. Ogata, in Strongly correlated electron systems II, ed. by G. Baskaran, A. E. Ruckenstein, E. Tosatti, and Yu Lu (World Scientific, Singapore, 1991), p. 31.

${ }^{43}$ K. A. Matveev, unpublished.

44 C. L. Kane and M. P. A. Fisher, Phys. Rev. B 46, 15233 (1992).

45 A. Furusaki and N. Nagaosa, Phys. Rev. B 47, 4631 (1993).

46 D. L. Maslov and M. Stone, Phys. Rev. B 52, R5539 (1995).

47 V. V. Ponomarenko, Phys. Rev. B 52, R8666 (1995).

48 I. Safi and H. J. Schulz, Phys. Rev. B 52, R17040 (1995).

49 L. B. Ioffe and A. I. Larkin, Phys. Rev. B 39, 8988 (1989).

${ }^{50}$ H. A. Bethe, Z. Phys. 71, 205 (1931).

51 E. Lieb and D. Mattis, Phys. Rev. 125, 164 (1962).

52 M. Karbach and G. Müller, Phys. Rev. B 62, 14871 (2000).

53 S. Frank, P. Poncharal, Z. L. Wang, and W. A. de Heer, Science, 280, 1744 (1998).

${ }^{54}$ L. D. Landau and E. M. Lifshitz, Quantum Mechanics, (Butterworth-Heinemann, Oxford, 1997), p. 183.

55 S. Coleman, Aspects of Symmetry, (Cambridge University Press, Cambridge, 1985), p. 341.

56 R. de Picciotto, H. L. Stormer, A. Yacoby, L. N. Pfeiffer, K. W. Baldwin, and K. W. West, Phys. Rev. Lett. 85 , 1730 (2000).

57 W. H. Furry, Phys. Rev. 71, 360 (1947).

58 M. Andrews, Am. J. Phys. 44, 1064 (1976).

59 M. M. Fogler, private communicaiton.

60 The numerical coefficients in the Hamiltonian (4) are different from those obtained by conventional bosonization $^{32.33}$ of the Heisenberg model. The discrepancy is due to the fact that the standard procedure uses different bosonic fields $\phi=\phi_{\sigma} / \sqrt{2}$ and $\theta=\sqrt{2} \theta_{\sigma}$.

61 One can easily check that the derivation of Eq. (9) is valid not only for a Wigner crystal, but for any translationinvariant model of interacting electrons.

62 The asymptotics at $x \rightarrow \pm \infty$ in Eq. 26) are chosen to correspond to outgoing plasmon wave.

63 Experiments with cleaved edge overgrowth wires,,$\frac{4}{-}$ show quantization below $2 e^{2} / h$, but this value is still independent of the electron density. The reduction of the quantized value was attributed to the imperfect coupling of the wire to the leads. 56

${ }^{64}$ It is worth mentioning that the violation of the spin-charge separation in Eq. (30) is due to the inhomogeneity of the system. In the homogeneous case $J=$ const, and the spincharge separation is restored.

65 The standard solution ${ }^{54}$ applies the WKB approximation to the ground states of the oscillatory potentials near the minima of $U(x)$. As a result it underestimates the level splitting (A4) by a factor ${ }^{57}$ of $\sqrt{\pi / e} \approx 1.075$. 